\title{
Crystal Structure of Photosystem I Monomer From Synechocystis PCC 6803
}

\author{
Sigal Yoli Netzer-El, Ido Caspy and Nathan Nelson*
}

Department of Biochemistry and Molecular Biology, The George S. Wise Faculty of Life Sciences, Tel Aviv University, Tel Aviv, Israel

A single histidine addition to the C-terminus of PsaL of Synechocystis sp. PCC 6803 was previously reported by our lab to shift the trimer-to-monomer ratio of PSI in favor of the monomeric form. P700 re-reduction and $\mathrm{NADP}^{+}$photo-reduction measurements of the PsaL ${ }^{H I S}$ strain show no effect on PSI activity in comparison to the WT trimeric PSI. Crystal structure of the PsaL ${ }^{\mathrm{HIS}}$ monomeric PSI reveals several alterations that occurred in the trimerisation site of PSI, primarily a deformation of the C-terminus of PsaL and loss of chlorophyll a and $\beta$-carotene molecules.

\section{OPEN ACCESS}

Edited by:

Henrik Scheller,

Lawrence Berkeley National Laboratory (LBNL), United States

Reviewed by:

Poul Erik Jensen, University of Copenhagen, Denmark Jean-David Rochaix,

Université de Genève, Switzerland

${ }^{*}$ Correspondence: Nathan Nelson nelson@post.tau.ac.i

Specialty section: This article was submitted to Plant Physiology,

a section of the journal Frontiers in Plant Science

Received: 26 September 2018 Accepted: 04 December 2018 Published: 04 January 2019

Citation:

Netzer-El SY, Caspy I and Nelson N (2019) Crystal Structure of Photosystem I Monomer From

Synechocystis PCC 6803.

Front. Plant Sci. 9:1865.

doi: 10.3389/fp/s.2018.01865
Keywords: cyanobacteria, membrane proteins, photosystem I (PSI), electron transport (photosynthetic), photosynthesis

\section{INTRODUCTION}

Oxygenic photosynthesis provides the necessary food and fuel for sustaining life on earth, by converting the sunlight energy into chemical energy available for all living creatures (Barber, 2004; Nelson, 2011). This intricate process takes place in photosynthetic organisms and is divided into a light dependent stage and a light independent stage. In the light dependent stage two large, multisubunit, pigment-protein complexes known as Photosystem I and II (PSI and PSII), are embedded in the thylakoid membranes, absorb light and turn physical energy into energy stored in chemical bonds. PSII mediates light-dependent water oxidation, which supplies electrons and protons to the photosynthetic process and releases oxygen, while PSI mediates NADP ${ }^{+}$photo-reduction (Nelson and Yocum, 2006).

A major portion of the world's photosynthesis occurs in the ocean and is performed by single celled mesophilic cyanobacteria and algae. The structure of both PSI and PSII was determined in thermophilic cyanobacteria at high resolution, 2.5 and $1.9 \AA$, respectively (Jordan et al., 2001; Umena et al., 2011). Recently our group published a high resolution structure of trimeric PSI at $2.5 \AA$ from the mesophilic cyanobacterium Synechocystis sp. PCC 6803 using x-ray crystallography (Malavath et al., 2018). This model shows the architecture of trimeric PSI (nearly 1 mega-Dalton) and consists of 33 protein subunits, 72 carotenoids, 285 chlorophyll a molecules, 51 lipids, 9 iron-sulfur clusters, 6 phylloquinones, and 6 putative calcium ions.

In cyanobacteria, PSI is found in a trimeric or monomeric form, though recently has been reported to appear also in a tetrameric form (Watanabe et al., 2011, 2014; Li et al., 2014; Semchonok et al., 2016). Under optimal growth conditions, the trimeric form of Synechocystis PSI is more abundant, whereas the monomeric form is present as a smaller fraction of the total complex. However, under different growth conditions, such as light, temperature and available nutrients the ratio of monomers to trimers may shift toward the monomeric form (Ivanov et al., 2006; Salomon and Keren, 2011; Kłodawska et al., 2014). The preference of one form over the other depending on 
environmental conditions suggests that each form functions slightly different, which may translate into changes in structure. Moreover, in our recently published PSI trimer model we showed a deviation from symmetry in which each monomer within the trimer holds a different set of ligands. It is still unclear whether the trimer is built from a homogenous pool of monomers that are modified later on to their specific ligand set, or three different monomer types are generated and then fused together to form the asymmetric trimer (Malavath et al., 2018).

To investigate the Synechocystis PSI monomer we performed a single histidine addition to the C-terminus of PsaL to generate a PsaL ${ }^{\text {HIS }}$ mutant strain (Malavath et al., 2018). The mutated strain generated mostly monomers (monomer-to-trimer ratio is 4.5:1), which allowed us to examine the monomer structure more easily, now constituting the major fraction of PSI, while taking specific interest in the trimerisation site. The crystals generated from PSI of PsaL ${ }^{\mathrm{HIS}}$ were solved to $4 \AA$ resolution and showed some differences in PSI structure, in comparison to WT PSI, primarily in the region of PsaL, and PsaI. Here we will present the structure of monomeric PSI and discuss the differences between the WT and the mutated PsaL ${ }^{\mathrm{HIS}}$ models.

\section{MATERIALS AND METHODS}

\section{PsaL ${ }^{\text {HIS }}$ Thylakoids Preparation and Monomer PSI Purification}

The PsaL ${ }^{H I S}$ construction, thylakoid preparation and PSI purification were described previously in Malavath et al. (2018). Briefly, PsaL ${ }^{\mathrm{HIS}}$ strain membranes were thawed and incubated with n-Dodecyl $\beta$-maltoside. After centrifugation, the sup was loaded onto a Toyopearl DEAE 650-C column. Green fractions were collected and precipitated with $100 \mathrm{mM} \mathrm{NaCl}$ and $10 \%$ PEG 3350. The re-suspension was loaded on a sucrose density gradient, dark green bands collected, and loaded on a SOURCE ${ }^{\mathrm{TM}}$ 15Q column. Dark green fractions were collected, loaded onto a sucrose density gradient and ultra-centrifuged. The dark green bands were collected from the gradients and used for crystallization. PSI trimer from the WT Synechocystis sp. PCC 6803 was purified by the standard procedure described in Malavath et al. (2018), except that only one column and one sucrose gradient were used during the purification. Briefly, WT strain membranes where thawed, and incubated with n-Dodecyl $\beta$-maltoside. After centrifugation, the sup was loaded onto a Toyopearl DEAE 650-C column. Green fractions collected and precipitated with $100 \mathrm{mM} \mathrm{NaCl}$ and 10\% PEG3350. The resuspension was loaded on a sucrose gradient, and dark green bands collected.

\section{PSI Crystallization and Cryogenic Protection}

Crystallization was carried out by the Sitting drop variant of the vapor-diffusion technique at $4^{\circ} \mathrm{C}$ (Charles Super Company). Aliquots $(4 \mu \mathrm{l})$ of PSI solution were mixed with equal volumes of a reservoir solution containing $160 \mathrm{mM} \mathrm{KCl,} 40 \mathrm{mM}$ MES$\mathrm{NaOH}$ (pH 6.5), and PEG 3350 (final content, 8-10\%). One of two additives was used to improve the resolution: either $0.01 \%$ sulfoquinovosyldiacylglycerol (SQDG, Avanti) or 5\% Jeffamine M-600 pH 7 (Hampton Research). Dark green crystals appeared after 3 days at the higher PEG concentrations, but the best diffracting crystals appeared after 2-3 weeks at the lower PEG concentrations. For cryogenic protection the crystals were soaked in a solution of $160 \mathrm{mM} \mathrm{KCl}, 40 \mathrm{mM}$ MES- $\mathrm{NaOH}$ ( $\mathrm{pH} 6.5$ ), and increasing PEG 3350 concentrations of 10, 15, 20, and 30\%. Crystals were sent for MS analysis to verify the presence of PsaL $^{\mathrm{HIS}}$, as described in Malavath et al. (2018).

\section{Data Collection and Processing}

The crystals were immediately frozen in liquid nitrogen. X-ray diffraction data were collected at the European Synchrotron Radiation Facility (ID23-2), the Swiss Light Source (PXI), and BESSYII (14.1). The data were analyzed, processed, and refined, as previously described by Mazor et al. (2014). Five data sets were combined using the AIMLESS suite for CCP4 to generate a $4 \AA$ dataset (Winn et al., 2011). STARANISO server was used for Anisotropicity corrections (Tickle et al., 2016).

\section{PSI Kinetic Measurements}

Plastocyanin of Synechocystis sp. PCC 6803 was heterologously expressed as described by Hulsker et al. (2007). The codonoptimized petE gene was delivered by IDT and ligated into expression vector pET17b (Invitrogen). The 28-amino acid transit peptide of petE was deleted and the following amino acid was replaced by a single methionine. Ferredoxin and FNR were produced and purified from Swiss chard (Nelson and Neumann, 1969; Yocum et al., 1975). Cytochrome c6 (cyt c533) was purified from Synechocystis (Mazor et al., 2014).

\section{P700 Reduction}

P700 reduction was measured in a Quartz cuvette, containing: $1 \mathrm{ml}$ reaction mix (20 mM Tricine- $\mathrm{NaOH}$ pH $8,5 \mathrm{mM} \mathrm{MgCl}_{2}$ and $0.05 \% \mathrm{n}$-Dodecyl $\beta$-maltoside), $10 \mu \mathrm{mol}$ Ascorbate, $100 \mathrm{nmol}$ Methyl viologen, $16 \mu \mathrm{g}$ chlorophyll PSI, and $50 \mathrm{pmol} \mathrm{Pc}$ of Synechocystis PCC SP 6803 or 50 pmol Cyt c 6 (Cyt C533). P700 photo-oxidation and re-reduction by $\mathrm{Pc}$ and $\mathrm{Cyt}_{\mathrm{C}} \mathrm{c}_{6}$ was measured by JTS-10 spectrophotometer by illuminating the sample with red light $(705 \mathrm{~nm})$ for $5 \mathrm{~s}$. Changes in absorbance were measured by $2 \mathrm{~ms}$ of LED light flashes $(700 \mathrm{~nm}$ passed through a $705 \mathrm{~nm}$ interference filter).

\section{NADP ${ }^{+}$Photo-Reduction Activity Assay}

In a Quartz cuvette, $1 \mathrm{ml}$ reaction mix $(20 \mathrm{mM} \mathrm{NaCl}, 10 \mathrm{mM}$ Tricine $\mathrm{NaOH}$ pH 8, $0.5 \mathrm{mM} \mathrm{MgCl}_{2}$ ) was supplemented with $20 \mu \mathrm{mol}$ ascorbate, $125 \mu \mathrm{g}$ Ferredoxin, $8.8 \mu \mathrm{g}$ FNR, $1 \mu \mathrm{mol}$ $\mathrm{NADP}^{+}$(Roche Diagnostics), $14 \mathrm{nmol} \mathrm{Pc}$, and PSI (14.4 $\mu \mathrm{g}$ chlorophyll). NADPH accumulation was measured at $340 \mathrm{~nm}$ $\left(\epsilon=6220 \mathrm{M}^{-1} \mathrm{~cm}^{-1}\right.$ ) by Cary 60 spectrophotometer (Agilent technologies) under continuous illumination with $660 \mathrm{~nm}$ LED light $(600 \mu \mathrm{E})$. The activity was calculated as $\mu \mathrm{mol} \mathrm{NADPH} /(\mathrm{mg}$ chl.Hr). 


\section{RESULTS}

As we previously reported, the addition of a single histidine at the C-terminus of PsaL (PsaL ${ }^{\mathrm{HIS}}$ ) was sufficient to turn the main fraction of PSI in the mutant strain from trimers to monomers caused by an interference to the trimer formation (Malavath et al., 2018). This allowed us to examine PSI monomers structure and function in comparison to PSI trimers.

\section{Kinetic Measurements}

Electron transport from PSI to ferredoxin was extensively studied (Munekage et al., 2004; Baniulis et al., 2008). Recently a crystal structure of trimeric PSI in complex with ferredoxin was published (Kubota-Kawai et al., 2018). The electron donation to oxidized P700 by reduced plastocyanin and cytochrome $\mathrm{c}_{6}$ was also studied using the trimeric wild-type PSI (Hippler et al., 1996). To compare the activity of WT PSI trimers and PsaL ${ }^{\mathrm{HIS}}$
PSI monomers we performed different kinetic tests. We measured P700 reduction rates by Plastocyanin and Cytochrome c6. In the presence of 50 pmol Pc, the re-reduction $\mathrm{t}_{1 / 2}$ of WT and PsaL ${ }^{\mathrm{HIS}}$ strains were $1.16 \pm 0.0001 \mathrm{~s}$ and $1.04 \pm 0.012 \mathrm{~s}$, respectively. In the presence of 50 pmol Cyt $\mathrm{C}_{6}$ the re-reduction $t_{1 / 2}$ were $1.33 \pm 0.014 \mathrm{~s}$ and $1.27 \pm 0.021 \mathrm{~s}$ for WT and PsaL ${ }^{\mathrm{HIS}}$ strains, respectively. All experiments were repeated three times $(n=3)$. Our results indicate that for both $\mathrm{Pc}_{\mathrm{c}}$ and Cyt $\mathrm{c}_{6}$, the $\mathrm{t}_{1 / 2}$ values of P700 re-reduction are very similar in WT PSI trimers and PsaL ${ }^{\mathrm{HIS}}$ PSI monomers (Figures 1A,B).

Moreover, we tested $\mathrm{NADP}^{+}$photo-reduction by PSI using saturated amounts of plastocyanin, ferredoxin and ferredoxinNADP-reductase, where ascorbate served as an electron donor. There was no significant difference in $\mathrm{NADP}^{+}$reduction rates between WT and PsaL ${ }^{\mathrm{HIS}}$ (323.24 $\pm 48.37 \mu \mathrm{mol} \mathrm{NADPH} /(\mathrm{mg}$ $\mathrm{chl} \cdot \mathrm{Hr})$ and $374.1 \pm 30.21 \mu \mathrm{mol} \mathrm{NADPH} /(\mathrm{mg} \quad \mathrm{chl} \cdot \mathrm{Hr})$, respectively) (Figure 1C).
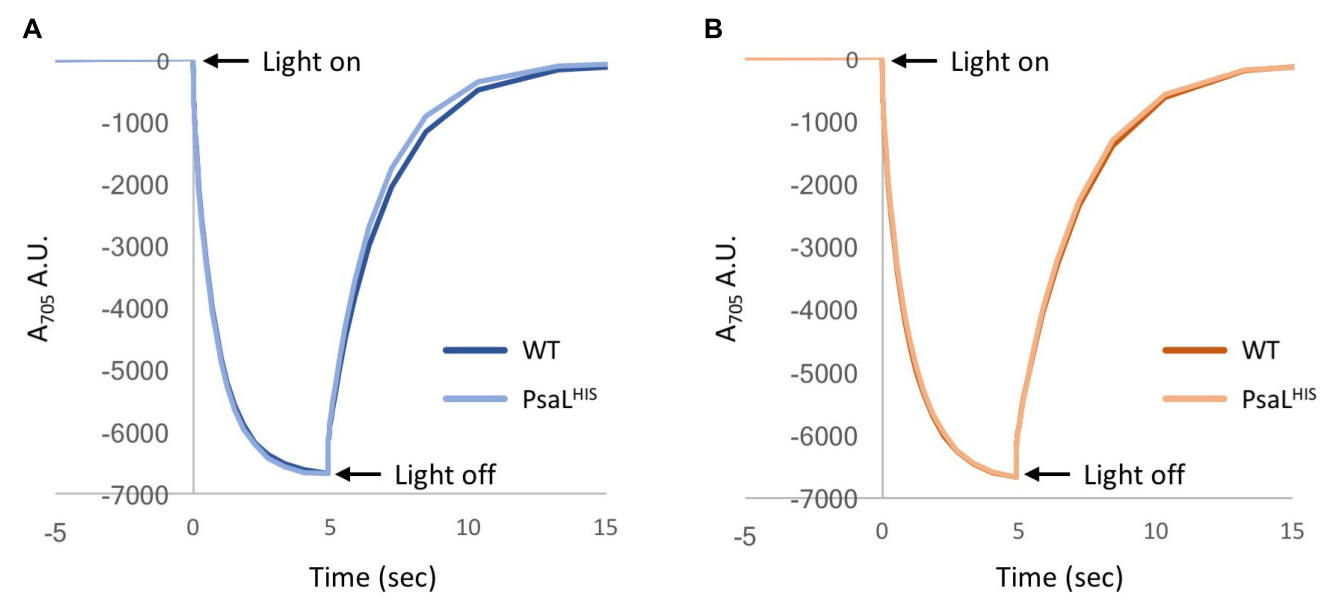

C

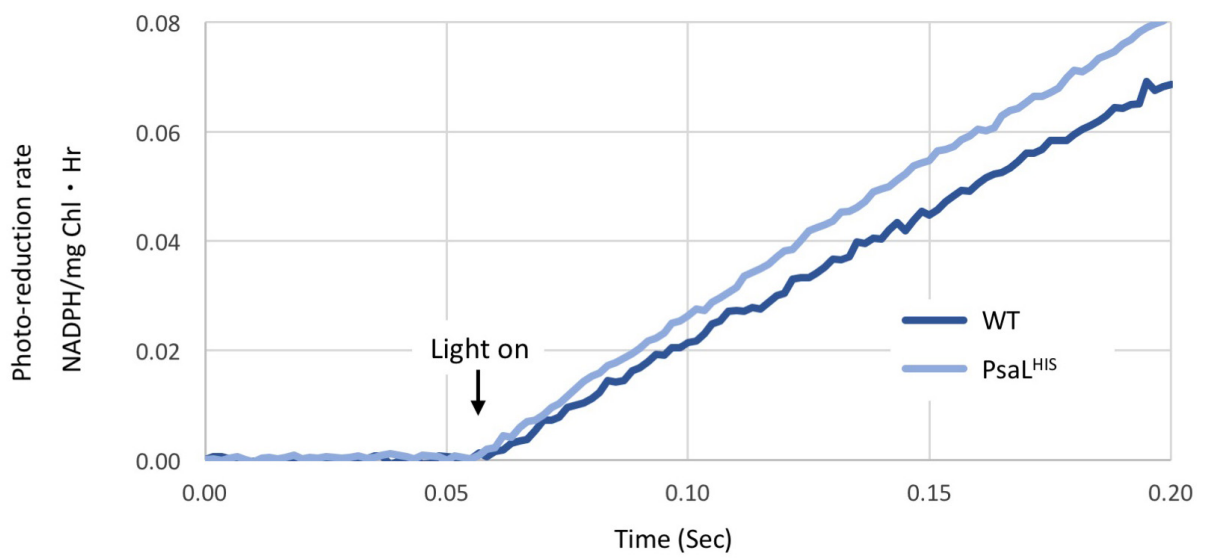

FIGURE 1 | Kinetic measurements of PSI from WT and PsaL HIS strains. P700 re-reduction - A reaction mix containing ascorbate and methyl viologen as electron source and terminal electron acceptor, respectively, was supplemented with $16 \mu \mathrm{g}$ Chl PSI and 50 pmol of either Plastocyanin (A) or Cytochrome $\mathrm{C}_{6}$ (B). The mix was illuminated with red light $(705 \mathrm{~nm})$ for $5 \mathrm{~s}$ and changes in absorbance were measured $(n=3)$. (A) Changes in $A_{705}$ in the presence of Pc, WT - dark blue; PsaL ${ }^{\text {HIS }}$ - light blue. (B) Changes in $\mathrm{A}_{705}$ in the presence of $\mathrm{Cyt}_{6} \mathrm{C}_{6}, \mathrm{WT}$ - dark orange; PsaL ${ }^{\text {HIS }}$ - light orange. (C) NADP+ photo-reduction rate - The electron transfer chain was reconstituted by mixing Pc, PSI, Fd, FNR, and NADP ${ }^{+}$. The mix was illuminated with $660 \mathrm{~nm}$ LED light (600 $\mu$ E) and NADPH accumulation was measured by absorbance at $340 \mathrm{~nm}$ by Cary 60 spectrophotometer $(n=3)$, WT - Dark blue; PsaL HIS - light blue. The calculate rate is shown as $\mu \mathrm{mol}$ NADPH/(mg chl. $\mathrm{Hr}$ ). 


\section{MAESNQVVQAYNGDPFVGHLSTPISDSAFTRT \\ FIGNLPAYRKGLSPILRGLEVGMAHGYFLIGP \\ WTLLGPLRDSEYQYIGGLIGALALILVATAAL \\ SSYGLVTFQGEQGSGDTLQTADGWSQFAAGFF \\ VGGMGGAFVAYFLLENLSVVDGIFRGLFNH*}

FIGURE 2 | PsaL ${ }^{\text {HIS }}$ identification by Mass-spectrometry. Presented is the amino acid sequence of subunit PsaL ${ }^{H I S}$; red - fragments of PsaL HIS identified by MS analysis.

\section{Achieving an Intact PSI Monomer Model}

The previous attempt to produce a crystal structure of PSI monomer from Synchocystis resulted in a PSI model lacking PsaI and PsaL, and therefore deficient of the trimerisation site (Mazor et al., 2014). In PsaL ${ }^{\mathrm{HIS}}$ a single histidine addition was presumed to cause a minimal disturbance to the complex's composition, thus allowing PSI monomer purification. Indeed, in the new

TABLE 1 | Statistics for the combined dataset of Synechocystis PSI PsaL ${ }^{\text {IIS }}$ monomer.

\begin{tabular}{ll}
\hline & Data collection \\
\hline Beamline & SLS X06SA PXI, ESRF ID23-2, BESSY II MX-14.1 \\
Wavelength $(\AA)$ & $0.999,0.873 .0 .918$ \\
Resolution $(\AA)$ & $49.5-4$ \\
Space group & P212121 \\
\hline
\end{tabular}

\begin{tabular}{lc}
\hline & Unit cell dimensions \\
\hline$a, b, c(\AA)$ & $124.32,178.66,181.45$ \\
$\alpha, \beta, \gamma$ & $90,90,90$ \\
Measured reflections & $839570(97420)$ \\
Unique reflections & $34852(4494)$ \\
Rpim $(\%)$ & $0.092(0.437)$ \\
$<\mathrm{I} / \sigma(I)>$ & $4.9(1.9)$ \\
CC1/2 & $0.983(0.759)$ \\
Completeness $(\%)$ & $99.7(98.5)$ \\
Redundancy & $24(21)$ \\
\hline & Refinement statistics \\
\hline Rwork/Rfree & $0.255 / 0.295$ \\
No. of chains & 11 \\
No. of ligands & 130 \\
Average B-factor $\left(\AA^{2}\right)$ & 126.2 \\
\hline
\end{tabular}

\begin{tabular}{lc}
\hline & R.M.S deviations \\
\hline Bond angles & 1.3 \\
Bond lengths & 0.004 \\
\hline & Ramachandran statistics \\
\hline Favored region (\%) & 91.45 \\
Allowed region (\%) & 7.6 \\
Outliers region (\%) & 0.95 \\
Clashscore & 14.6
\end{tabular}

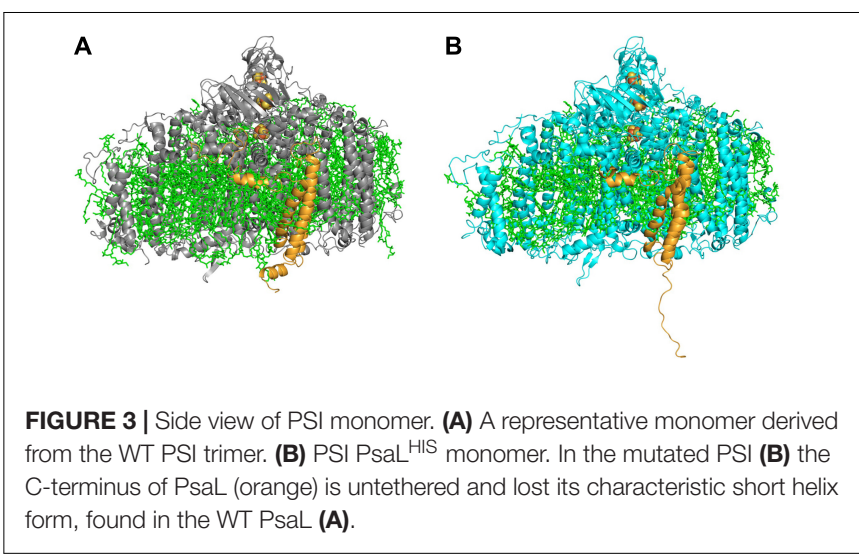

PsaL $^{\text {HIS }}$ strain the PSI trimer fraction has decreased considerably and the presence of PsaL in the complex monomers was verified by MS (Figure 2).

After screening hundreds of PsaL ${ }^{\mathrm{HIS}}$ PSI crystals, we collected data sets from a few dozen crystals which diffracted to low resolution. When examined, none of the data sets could be used individually to produce an adequate model due to poor signal to noise ratio, variations in unit cell values and high anisotropicity.

Using AIMLESS we were able to combine five individual datasets to ultimately create a $4 \AA$ dataset (Winn et al., 2011). Anisotropicity was still a major problem after combining the sets, so it was corrected in the unified dataset by the STARANISO server (Tickle et al., 2016). ${ }^{1}$ These crystals had a P212121 symmetry and have all displayed a similar unit cell dimensions of a 124.32, b 178.66, c 181.45, and $\alpha, \beta, \gamma$ 90, 90, 90 on average. Table 1 shows the $\mathrm{x}$-ray collection and refinement statistics, prior to the anisotropic correction applied on the dataset. The map and model are available in the PDB under the ID 6HQB.

\section{Crystal Structure of PsaL ${ }^{\text {HIS }}$ Monomer}

Cyanobacterial PSI is usually found in two oligomeric states trimeric and monomeric, though a tetrameric form has been demonstrated (Watanabe et al., 2011; Li et al., 2014; Semchonok et al., 2016). In the WT strain of Synechocystis PSI is mainly found in the trimeric form. We demonstrated that upon addition of a single histidine at the C-terimnus of PsaL, the dominant form of PSI super-complex was altered from a trimer to a monomer (Figure 3) (Malavath et al., 2018).

As could be expected, the most drastic changes occurred in the structure and associated ligands of PsaL. While the wildtype PsaL coordinates three chlorophyll a molecules (L1501, L1502 and L1503) and two $\beta$-carotenes (L4019 and L4022), only chlorophyll L1502 was modeled into the electron density map of PsaL ${ }^{\text {HIS }}$ monomer. $\beta$-carotene L4022 is located outside the PSI cylinder and in the trimeric structure it is wedged between chlorophylls L1501 and L1503 and two neighboring PsaL subunits, possibly binding them together. PsaL's second $\beta$ carotene, L4019, is encompassed on all sides by chlorophylls L1502 and B1239 on one end and PsaL and PsaI on the outer

\footnotetext{
${ }^{1}$ http://staraniso.globalphasing.org/cgi-bin/staraniso.cgi
} 

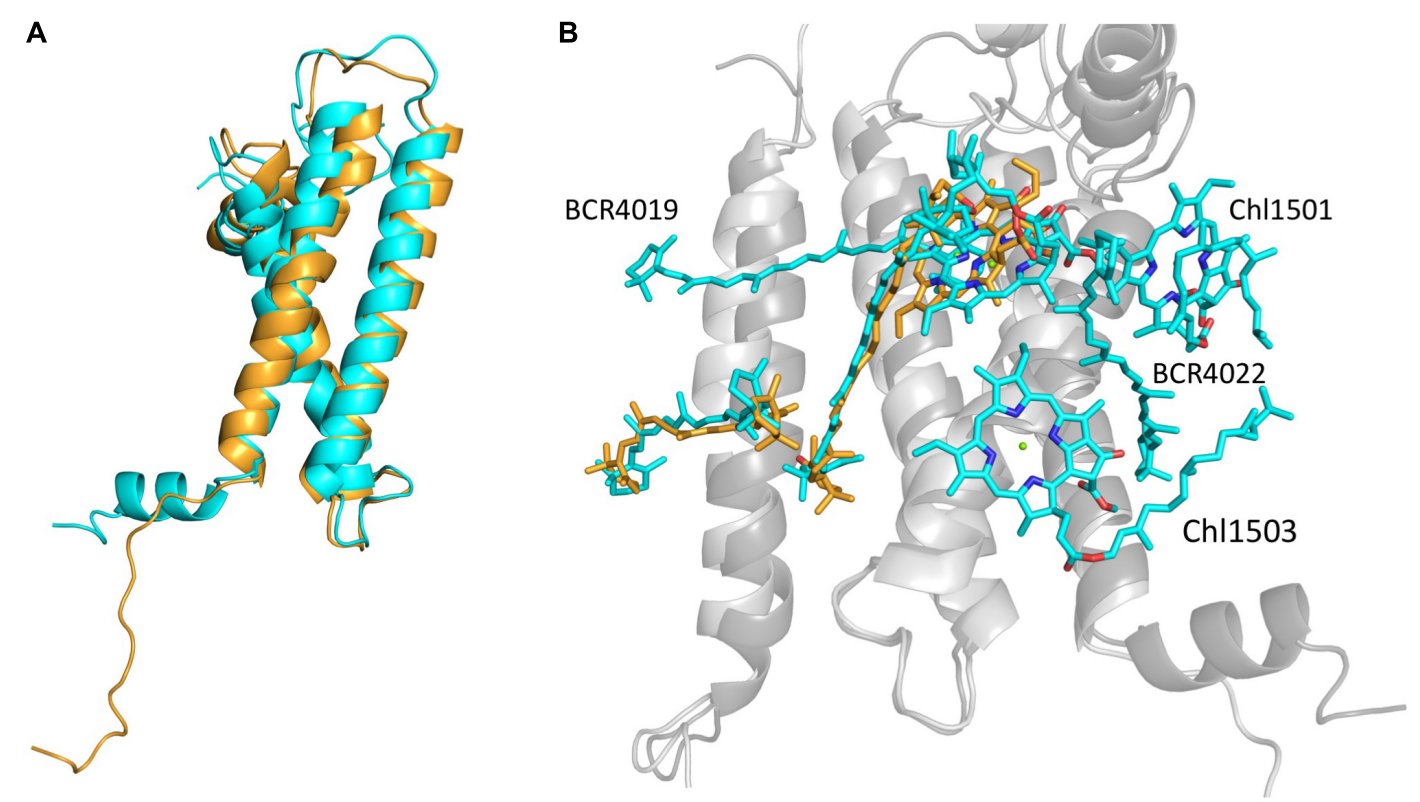

FIGURE 4 | (A) Backbones of PsaL HIS (orange) and trimer PsaL (cyan) alignment. (B) PsaL, Psal and their ligands from PsaL ${ }^{\text {HIS }}$ (backbone - light gray; ligands orange) are aligned to their correspondents from trimeric PSI (backbone - dark gray; ligands - cyan). The PsaL ${ }^{\text {HIS }}$ model is missing 2 Chlorophyll a molecules and 2 $\beta$-carotenes present in the trimer model.

edge. Both chlorophyll a molecules L1501 and L1503, located at the outskirts of PsaL and coordinated by its first helix. It is reasonable to assume that any shift in the location of PsaL, which was observed in our monomeric structure, could interfere with ligand binding or prevent it entirely (Figure 4B).

The C-terminus region of the PsaL in the native trimeric PSI (ASN144-ASN157) creates a short helix which can interact with the neighbor monomer's PsaL and assist in trimerisation stabilization, however, the addition of a terminal histidine has disrupted the short helical structure and created a loose C-terminus in PsaL (Figure 4A).

The largest dislocation and structural differences in the PSI subunits was observed in PsaM, PsaI and PsaL which are all located at the trimerisation area. Despite a shift of 2-3 $\AA$ in PsaI location toward PsaM and away from PsaL, both carotenoids that were coordinated by this subunit remained in their position and orientation. However, a dislocation of about $1 \AA$ in PsaM from its original position caused carotenoid M4021 to interact exclusively with PsaM whereas in the native form it is coordinated by both PsaM and PsaI.

\section{DISCUSSION}

PSI has been extensively studied for many years in hope of elucidating the way by which it converts sunlight to chemical energy (Grotjohann and Fromme, 2005; Qin et al., 2015). Previous studies demonstrated that inactivation of PsaI or PsaL genes in S. 6803 resulted in the absence of PsaL from the PSI complex in S. 6803. These studies showed the importance of PsaI in PsaL's integration into the complex and PsaL's role in PSI trimerisation (Chitnis et al., 1993; Xu et al., 1995).
The goal of this project was to determine which differences if any, exist in the structure or function between PSI monomers and trimers. To that end we created a new Synechocystis S.6803 strain, $\mathrm{PsaL}^{\mathrm{HIS}}$, in which the monomer is the larger fraction.

Structurally, several changes were observed at the trimerisation region of the complex. In the WT PsaL, the C-terminus is fixed in a compact $\alpha$-helix form, however, in $\mathrm{PsaL}^{\mathrm{HIS}}$ it is untethered. The addition of a single histidine at the end of the sequence is not expected to interrupt the formation of the protein secondary structure. This emphasizes the importance of PsaL's C-terminus in PSI trimerisation, as it has to maintain the integrity of its secondary structure in order for trimerisation to occur. Moreover, the WT PsaL contains three chlorophyll a molecules and two $\beta$-carotenes, whereas the PsaL ${ }^{\mathrm{HIS}}$ monomer contains only one chlorophyll a molecule and no $\beta$-carotene molecules. It is possible that the presence of these ligands contribute to the structural stability that holds the C-terminus in its place. An alternative hypothesis is that the lack of stable secondary or tertiary structures prevents the integration of the ligands into the protein. Whichever the case may be, the results suggest that these ligands, that are possibly incorporated during trimerisation, might play a structural role and not just participate in excitation energy transfer dissipation.

Despite the change to PSI super complex's oligomeric state in the new PsaL ${ }^{\mathrm{HIS}}$ strain, the kinetic tests we preformed showed no significant effect on the activity of PSI. This finding coincides with the structure obtained from the mutant crystals, which shows no critical changes in the complex with the exception of the trimerisation site.

In the recently published WT PSI model, we reported that PsaL also hold a putative $\mathrm{Ca}^{2+}$ ion and several phospholipids 
(Malavath et al., 2018). We suggested that this $\mathrm{Ca}^{2+}$ ion contributes to the trimer formation and stabilization by holding two neighboring PsaL subunits together.

Our limited resolution does not allow to ascertain that the ligands mentioned had not been incorporated to the structure, or that they had diffused out of the structure since they were located at the outskirts of the super-complex. Although our low resolution structure does not permit us to identify the exact ligand composition of the $\mathrm{PsaL}^{\mathrm{HIS}}$ monomer, we can assume there is a specific set of ligands that defines the main monomer. This putative monomer is used to assemble either the trimeric PSI or modified to create other asymmetric monomers for which our model can be used as a representative.

\section{DATA AVAILABILITY STATEMENT}

The following dataset was generated:

\begin{tabular}{lllc}
\hline Author(s) & Year & Dataset title & Dataset ID \\
\hline Netzer-El S.Y. & 2018 & $\begin{array}{l}\text { Monomeric } \\
\text { cyanobacterial } \\
\text { Caspy I }\end{array}$ & PDB ID 6HQB \\
Nelson N & & & \\
\hline
\end{tabular}

\section{REFERENCES}

Baniulis, D., Yamashita, E., Zhang, H., Hasan, S. S., and Cramer, W. A. (2008). Structure-function of the cytochrome b6f complex. Photochem. Photobiol. 84, 1349-1358. doi: 10.1111/j.1751-1097.2008.00444.x

Barber, J. (2004). Engine of life and big bang of evolution: a personal perspective. Photosynth. Res. 80, 137-155. doi: 10.1023/B:PRES.0000030662.04618.27

Chitnis, V. P., Xu, Q., Yu, L., Golbeck, J. H., Nakamoto, H., Xie, D. L., et al. (1993). Targeted inactivation of the gene psaL encoding a subunit of photosystem I of the cyanobacterium Synechocystis sp. PCC 6803. J. Biol. Chem. 268, 1167811684.

Grotjohann, I., and Fromme, P. (2005). Structure of cyanobacterial photosystem I. Photosynth. Res. 85, 51-72. doi: 10.1007/s11120-005-1440-4

Hippler, M., Reichert, J., Sutter, M., Zak, E., Altschmied, L., Schröer, U., et al. (1996). The plastocyanin binding domain of photosystem I. EMBO J. 15, 6374-6384. doi: 10.1002/j.1460-2075.1996.tb01028.x

Hulsker, R., Mery, A., Thomassen, E. A., Ranieri, A., Sola, M., Verbeet, M. P., et al. (2007). Protonation of a histidine copper ligand in fern plastocyanin. J. Am. Chem. Soc. 129, 4423-4429. doi: 10.1021/ja0690464

Ivanov, A. G., Krol, M., Sveshnikov, D., Selstam, E., Sandström, S., Koochek, M., et al. (2006). Iron deficiency in cyanobacteria causes monomerization of photosystem I trimers and reduces the capacity for state transitions and the effective absorption cross section of photosystem I in vivo. Plant Physiol. 141, 1436-1445. doi: 10.1104/pp.106.082339

Jordan, P., Fromme, P., Witt, H. T., Klukas, O., Saenger, W., and Krauss, N. (2001). Three-dimensional structure of cyanobacterial photosystem I at $2.5 \mathrm{~A}$ resolution. Nature 411, 909-917. doi: 10.1038/35082000

Kłodawska, K., Kovács, L., Várkonyi, Z., Kis, M., Sozer, Ö, Laczkó-Dobos, H., et al. (2014). Elevated growth temperature can enhance photosystem I trimer formation and affects Xanthophyll biosynthesis in cyanobacterium Synechocystis sp. PCC6803 cells. Plant Cell Physiol. 56, 558-571. doi: 10.1093/ pcp/pcu199

Kubota-Kawai, H., Mutoh, R., Shinmura, K., Sétif, P., Nowaczyk, M. M., Rögner, M., et al. (2018). X-ray structure of an asymmetrical trimeric ferredoxin-photosystem I complex. Nat. Plants 4, 218-224. doi: 10.1038/ s41477-018-0130-0

\section{AUTHOR CONTRIBUTIONS}

$\mathrm{SN}-\mathrm{E}$ performed the biochemical procedures, isolation of PSI, crystallization, data collection, kinetic measurements, discussion, and writing the paper. IC performed the structure determination, data collection, discussion, and writing the paper. $\mathrm{NN}$ supervised the study, collected the data, and participated in the experiments.

\section{FUNDING}

This work was supported by grant (No. 293579 - HOPSEP) from the European Research Council, by The Israel Science Foundation (Grant No. 569/17), and by the I-CORE Program of the Planning and Budgeting Committee and The Israel Science Foundation (Grant No. 1775/12).

\section{ACKNOWLEDGMENTS}

The authors would like to thank the ESRF, SLS, and BESSYII synchrotrons for beam time and the staff scientists for excellent guidance and assistance.

Li, M., Semchonok, D. A., Boekema, E. J., and Bruce, B. D. (2014). Characterization and evolution of tetrameric photosystem I from the thermophilic cyanobacterium Chroococcidiopsis sp. TS-821. Plant Cell 26, 1230-1245. doi: 10.1105/tpc.113.120782

Malavath, T., Caspy, I., Netzer-El, S. Y., Klaiman, D., and Nelson, N. (2018). Structure and function of wild-type and subunit-depleted photosystem I in Synechocystis. Biochim. Biophys. Acta 1859, 645-654. doi: 10.1016/j.bbabio. 2018.02.002

Mazor, Y., Nataf, D., Toporik, H., and Nelson, N. (2014). Crystal structures of virus-like photosystem I complexes from the mesophilic cyanobacterium Synechocystis PCC 6803. eLife 3:e01496. doi: 10.7554/eLife.01496

Munekage, Y., Hashimoto, M., Miyake, C., Tomizawa, K., Endo, T., Tasaka, M., et al. (2004). Cyclic electron flow around photosystem I is essential for photosynthesis. Nature 429, 579-582. doi: 10.1038/nature 02598

Nelson, N. (2011). Photosystems and global effects of oxygenic photosynthesis Biochim. Biophys. Acta 1807, 856-863. doi: 10.1016/j.bbabio.2010. 10.011

Nelson, N., and Neumann, J. (1969). Interaction between ferredoxin and ferredoxin-NADP reductase in pyridine nucleotide photoreduction and some partial reactions. I. Inhibition of ferredoxin-NADP reductase by ferredoxin. J. Biol. Chem. 244, 1926-11931.

Nelson, N., and Yocum, C. F. (2006). Structure and function of photosystems I and II. Annu. Rev. Plant Biol. 57, 521-565. doi: 10.1146/annurev.arplant.57.032905. 105350

Qin, X., Suga, M., Kuang, T., and Shen, J. R. (2015). Photosynthesis. Structural basis for energy transfer pathways in the plant PSI-LHCI supercomplex. Science 348, 989-995. doi: 10.1126/science.aab0214

Salomon, E., and Keren, N. (2011). Manganese limitation induces changes in the activity and in the organization of photosynthetic complexes in the cyanobacterium Synechocystis sp. Strain PCC 6803. Plant Physiol. 155, 571-579. doi: 10.1104/pp.110.164269

Semchonok, D. A., Li, M., Bruce, B. D., Oostergetel, G. T., and Boekema, E. J. (2016). Cryo-EM structure of a tetrameric cyanobacterial photosystem I complex reveals novel subunit interactions. Biochim. Biophys. Acta 1857, 1619-1626. doi: 10.1016/j.bbabio.2016.06.012 
Tickle, I. J., Bricogne, G., Flensburg, C., Keller, P., Paciorek, W., Sharff, A., et al. (2016). STARANISO. Cambridge: Global Phasing Ltd.

Umena, Y., Kawakami, K., Shen, J. R., and Kamiya, N. (2011). Crystal structure of oxygen-evolving photosystem II at a resolution of $1.9 \AA$ A Nature 473, 55-60. doi: 10.1038/nature09913

Watanabe, M., Kubota, H., Wada, H., Narikawa, R., and Ikeuchi, M. (2011). Novel supercomplex organization of photosystem I in anabaena and Cyanophora paradoxa. Plant Cell Physiol. 52, 162-168. doi: 10.1093/pcp/pcq183

Watanabe, M., Semchonok, D. A., Webber-Birungi, M. T., Ehira, S., Kondo, K., Narikawa, R., et al. (2014). Attachment of phycobilisomes in an antenna-photosystem I supercomplex of cyanobacteria. Proc. Natl. Acad. Sci. U.S.A. 111, 2512-2517. doi: 10.1073/pnas.13205 99111

Winn, M. D., Ballard, C. C., Cowtan, K. D., Dodson, E. J., Emsley, P., Evans, P. R., et al. (2011). Overview of the CCP4 suite and current developments. Acta Crystallogr. D Biol. Crystallogr. 67, 235-242. doi: 10.1107/S090744491004 5749

Xu, Q., Hoppe, D., Chitnis, V. P., Odom, W. R., Guikema, J. A., and Chitnis, P. R. (1995). Mutational analysis of photosystem I polypeptides in the cyanobacterium Synechocystis sp. PCC 6803. Targeted inactivation of psaI reveals the function of psaI in the structural organization of psaL. J. Biol. Chem. 270, 16243-16250. doi: 10.1074/jbc.270.27. 16243

Yocum, C. F., Nelson, N., and Racker, E. (1975). A combined procedure for preparation of plastocyanin, ferredoxin and CF1. Prep. Biochem. 5, 305-317. doi: 10.1080/00327487508061579

Conflict of Interest Statement: The authors declare that the research was conducted in the absence of any commercial or financial relationships that could be construed as a potential conflict of interest.

Copyright (c) 2019 Netzer-El, Caspy and Nelson. This is an open-access article distributed under the terms of the Creative Commons Attribution License (CC BY). The use, distribution or reproduction in other forums is permitted, provided the original author(s) and the copyright owner(s) are credited and that the original publication in this journal is cited, in accordance with accepted academic practice. No use, distribution or reproduction is permitted which does not comply with these terms. 